\title{
Characterization and Fatigue of the Converse Piezoelectric Effect in PZT Films for MEMS Applications
}

\author{
Andrea Mazzalai, Davide Balma, Nachiappan Chidambaram, Ramin Matloub, and Paul Muralt, Fellow, IEEE
}

\begin{abstract}
A measurement setup for the detailed study of the transverse piezoelectric coefficient $e_{31, f}$ in the converse (actuator) mode was developed. It allows the assessment of the piezoelectric stress in thin films on silicon cantilevers and provides for a correlation of this stress with large and small signal responses to ferroelectric polarization and dielectric response, both as a function of slowly sweeping electric field. This test is important for the understanding of piezoelectric thin films in microelectromechanical systems. The method is illustrated at hand of sol-gel lead-zirconate-titanate (PZT) thin films, and verified also with AIN and AIN-ScN alloy thin films. A $1-\mu \mathrm{m}$ thick, sol-gel derived PZT(53/47) gradient-free sample showed a response of $-18.3 \mathrm{C} / \mathrm{m}^{2}$ at $100-\mathrm{kV} / \mathrm{cm}$ electric field. Reliability tests of PZT thin films were carried out with the same setup in an accelerated manner. The piezoelectric activity did not degrade significantly up to $10^{9}$ unipolar pulses at $100 \mathrm{kHz}$ with an amplitude of $-150 \mathrm{kV} / \mathrm{cm}$. The increase in leakage toward the end of the cycles was explained by a thermal runaway effect.

[2014-0140]
\end{abstract}

Index Terms-Piezoelectric, actuator, setup, $e_{31, f}$, stress, reliability, cantilever, displacement, lead-zirconate-titanate (PZT), aluminum nitride.

\section{INTRODUCTION}

$\mathbf{T}$ HE RECENT progress in synthesis and integration of lead-zirconate-titanate (PZT) thin films onto silicon substrates, combined with increasing interests and efforts of industry, is paving the way for new, more efficient and faster devices in micro electro-mechanical systems (MEMS) through the use of the piezoelectric effect in these films. Very promising markets are predicted for the so-called piezo-MEMS technology. The high piezoelectric activity of these materials is such that, in many cases, piezoelectric actuation offers more freedom of design, needs less voltage (as compared to electrostatic devices) or less power (as compared to thermal actuators), and exhibits larger strokes per electrical energy input. The most promising high-volume products are faster and more precise ink-jet print-heads [1], faster and less power consuming autofocus lenses, and a plethora of other devices, such as

Manuscript received April 30, 2014; revised August 17, 2014; accepted August 26, 2014. This work was supported in part by the Swiss National Science Foundation under Contract FN 200020-138154, and in part by the Swiss Commission for Technology and Innovation under Contract 15461.1. Subject Editor G. Piazza.

The authors are with the Department of Materials Science, École Polytechnique Fédérale de Lausanne, Lausanne CH-1015, Switzerland (e-mail: andrea.mazzalai@gmail.com; davide.balma@epfl.ch; nachiappan. chidambaram@epfl.ch; ramin.matloub@epfl.ch; paul.muralt@epfl.ch).

Color versions of one or more of the figures in this paper are available online at http://ieeexplore.ieee.org.

Digital Object Identifier 10.1109/JMEMS.2014.2353855 micro-pumps [2], low-voltage micromotors [3], piezoelectric microphones [4], photoacoustic detectors [5], micromachined ultrasound transducers (pMUTs) [6], RF switches [7], [8], robotic micro-legs [9], resonant scanners for high-definition projectors [10], and many more. The technology is even studied for the realization of flying robots with wings [11], [12]. Most of these devices are actuators, into which parallel plate capacitor structures with PZT thin films are integrated. Their functionality relies on the effective transverse piezoelectric effect, i.e. the development of an in-plane piezoelectric stress upon application of an electric field. The latter is used to bend a flexural structure in this actuator mode. A piezoelectric thin film is clamped on the substrate and free to expand in the out-of-plane direction. By applying these boundary conditions to the constitutive equations of piezoelectricity, it is found that the in-plane stress $\sigma_{1}$ is generated by the piezoelectric coefficient $e_{31, f}=-\sigma_{1} / E_{3}=d_{31} /\left(s_{11}+s_{12}\right)=e_{31}-e_{33}\left(c_{13} / c_{33}\right)$, which is larger than the fully clamped $e_{31}$ [13]. PZT is in addition a ferroelectric material; therefore the polarization, and, as a consequence, the piezoelectric properties, vary with the voltage. As the film thicknesses are small, electric fields are large enough to make the non-linearity of the piezoelectric activity visible. In other terms, the piezoelectric coefficients are not constant and therefore a single number cannot describe in details the electromechanical behavior of the system.

Efforts have been put to measure the effective transverse piezoelectric activity of thin films in a standardized way. Shepard et al. [14] established a setup that bends the whole wafer by applying a pressure difference between the front and the backside. The resulting deformation is measured through strain gauges placed on the surface of the sample, nearby the test electrodes which are used to detect piezoelectric charges [15]. Dubois et al. [16] proposed a setup with fullwafer thickness cantilevers ( $1.5 \mathrm{~mm}$ by $15 \mathrm{~mm}$ in size) that are clamped at one end and bent through a calibrated actuator at the other end. With this geometry, the in-plane strain in the area covered by the top electrode is not quite uniform, and thus a short electrode is chosen to measure the effect. The varying curvature is taken into account in the mathematical description, which is based on the geometry of the sample, the tip position and its displacement. The direct $e_{31, f}$ is then determined from the measured piezoelectric charges. As the unknown film is much thinner than the (homogeneous) cantilever, this bending method needs only one material constant: the Poisson's ratio of the substrate. This method has been improved in 2007 in the aixACCT's commercial setup with the introduction of the 
four-point bending technique [17]: a full-wafer thickness cantilever is placed between four cylindrical rods. The outer two are both loaded with a mechanical force and are simultaneously used to contact the sample electrodes. If the inner supports (which are placed on the opposite side of the substrate) are spaced by half the distance respect to the outer ones, the mechanical force induces a constant bending moment on the cantilever, which translates into a homogeneous stress and strain distribution between the inner supports. By measuring the output charges and the displacement at the center of the top electrode, $e_{31, f}$ can be calculated. The setups described above reveal only the direct piezoelectric activity. Although essential for sensors or energy harvesting applications, these measurements can only provide information about the remanent values, leaving unknowns on the film response at higher driving voltages. For actuators indeed, relevant $e_{31, f}$ values need to be known for higher electric fields, and in addition for specific voltage cycles. A convenient way to evaluate the transverse converse effective piezoelectric activity of a thin film is to track the tip displacement of a full wafer thickness cantilever structure as a function of the externally applied electric field. This geometry is particularly useful for two main reasons. First of all the sample preparation does not need advanced micromachining steps, as just top electrode patterning with a stencil and dicing are required. The same samples can be used to evaluate the direct effect with the setups described above. Second, as we will show, the in-plane stress in the film can be derived analytically, if the sample geometry and the elastic properties of the substrate are known, and independently on the elastic properties of the film since it is much thinner than the substrate. An evaluation of the active-mode $e_{31, f}$, based on a cantilever tip displacement measurements, has been recently reported by Chun, Sato and Kanno [18]. In their case the cantilever has also the full-wafer thickness, but the electrode covers completely the surface. Unfortunately in their paper no in-plane stress/strain function is reported as a function of the applied electric field: the analysis is limited to an average $e_{31, f}$ determination from the ratio of the large-signal amplitudes of displacement and electric field. The so-obtained value is not exhaustive for the characterization of the film as, for example, the amount of hysteresis (which is always present in ferroelectrics, even in unipolar excitation) remains unknown. A more suitable evaluation of the transverse converse piezoelectric coefficient for actuator applications is represented instead by the derivative of the in-plane stress function:

$$
e_{31, f}\left(E_{3}\right)=-\frac{d \sigma_{1}}{d E_{3}}
$$

With this derivative function we can describe the piezoelectric response and its hysteresis under arbitrary excitation conditions. In order to characterize in a satisfactory way the piezoelectric activity of a film, at least the values obtained upon increasing and decreasing electric fields at half excitation amplitude should be reported, in order to give an idea about the extent of the hysteresis. If desired, the average between them might be used as a representative value for the film. Moreover the polarization hysteresis loop and the small-signal
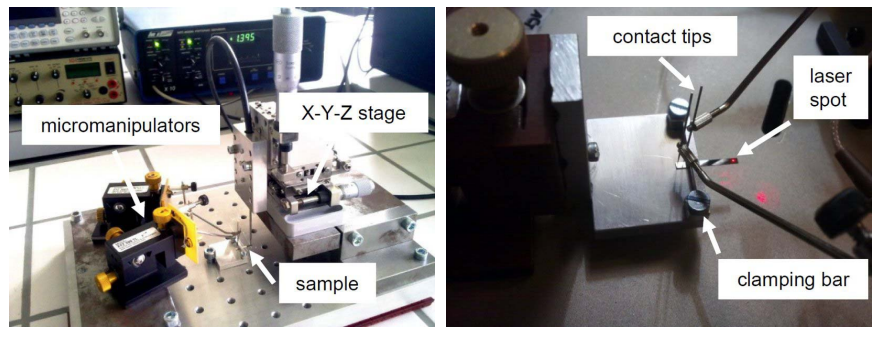

Fig. 1. Pictures of the developed setup. On the left in the optical probe version; while on the right we can appreciate a zoom of the sample in the single-laser beam interferometer version.

$\mathrm{C}-\mathrm{V}$ loop can in principle be recorded simultaneously with the piezoelectric response, leading to an accurate evaluation of the film electromechanical behavior under excitation conditions mimicking as close as possible the ones desired for piezo-MEMS devices. For instance, the P-V loop carries in its integral the energy dissipated during one excitation cycle. Such information might be as important as the knowledge of the piezoelectric coefficients. The industrialization of a piezo-MEMS product needs moreover preliminary information on reliability of the film itself upon repeated voltage cycles. Lifetime tests, although accelerated, need to be performed at conditions as close as possible to the relevant ones of the application. For these reasons we developed and tested a cheap and easy-to-use setup to evaluate in details the piezoelectric and dielectric properties of ferroelectric thin films for piezo-MEMS actuators.

\section{Measurement Setup}

\section{A. Description of the Apparatus}

Expected tip displacements for millimeter long cantilevers are in a range between fractions of nanometers for small signal excitations of AlN thin films, to several hundreds of nanometers for large signal response of PZT thin films. Such movements can be tracked either by a single-laser beam Michelson interferometer, or with a proximity sensor. A detailed analysis of the principles and a quantitative analysis of the performance characteristics of this type of transducer has been performed by Cook and Hamm [19]. Commercial instruments are available for both single-beam interferometry and proximity sensing. Results discussed here are obtained either with a SIOS ${ }^{\circledR}$ SP-S laser interferometer or with an MTI $^{\circledR} 2000$ fotonic sensor. For the latter we measured a sensitivity of $5.90 \pm 0.07 \mu \mathrm{m} / \mathrm{V}$, a noise level equivalent to displacements of $2 \mathrm{~nm}$, and a measurement range $30 \mu \mathrm{m}$ wide.

Pictures of the developed setup are shown in Fig. 1. In the optical probe version, the displacement sensor is mounted on an X-Y-Z stage controlled by micrometer screws drives, allowing for precise positioning of the measurement head and calibration of the sensor on the fly. For the laser beam based setup, the sensor is instead fixed, and the sample is moved during the alignment procedure. In both cases the top and bottom electrodes are accessed via micro-manipulators.

The operating principle is depicted in Fig. 2. A fullwafer thickness cantilever is clamped with a $2 \mathrm{~mm}$ wide and 


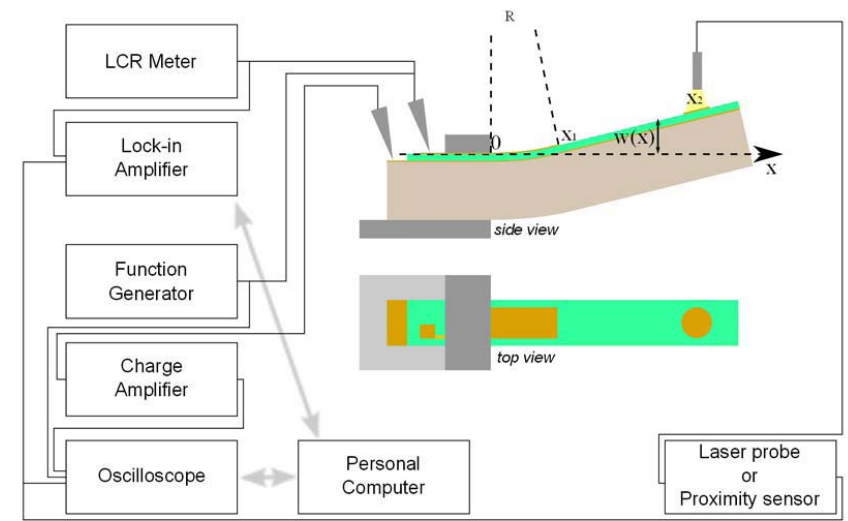

Fig. 2. Scheme of the cantilever-tip displacement measurement setup developed for the simultaneous measurement of the piezoelectric and ferroelectric properties of the sample. In the sector between the clamp at $x=0$ and the top electrode end at $x=x_{1}$ the cantilever shows a constant curvature assuming a homogeneous $e_{31, f}$. The deflection is exaggerated for clarification purposes.

$3 \mathrm{~mm}$ thick bar onto a plate, both made of stainless steel. The beam is excited either with an LCR meter (for small-signal measurements) or with a function generator (for arbitrary input waveform). In the sector between the clamp and the top electrode end, the beam will be deformed by the piezoelectric activity of the film. If it is uniform, the radius of curvature is constant. In the sector uncovered by the top electrode, there is no bending of the cantilever: the slope remains constant, and the deflection just increases linearly with the distance from the electrode end. The displacement at the tip is tracked with the help of a lock-in amplifier for small-signal excitations, or directly with an oscilloscope for large-signal ones. In this case the bottom electrode can be contacted with a charge amplifier (an operational amplifier with a capacitor in the feedback integrating the current) to measure simultaneously the polarization hysteresis loops. Results shown are acquired either with the fotonic sensor coupled with an HP4284A LCR meter and a Kistler ${ }^{\circledR}$ Type 5011 charge amplifier, or with the single-laser beam interferometer coupled with an aixACCT ${ }^{\circledR}$ TF2000 setup.

\section{B. Analytical Expression for the In-Plane Stress}

We now derive the analytical expression which links the in-plane stress $\sigma_{1}$ to the tip displacement. Given that the substrate is much thicker than the film, the radius of curvature $R$ in the area below the electrode will be large (small deflection). We can then state

$$
\frac{1}{R}=\frac{d^{2} w(x)}{d x^{2}}
$$

where $w(x)$ is the cantilever deflection function along the longitudinal axis as a function of the distance from the clamp (positioned at $x=0$ ). The beam will not be bent in the sector uncovered by the top electrode: from its end $\left(x=x_{1}\right)$ onwards the derivative of the displacement function is constant. At the probe position $\left(x=x_{2}\right)$ we have then an amplification of the displacement given by the derivative $w\left(x_{1}\right)^{\prime}$ multiplied by the distance $x_{2}-x_{1}$ :

$$
w\left(x_{2}\right)=w\left(x_{1}\right)+\left(x_{2}-x_{1}\right) w\left(x_{1}\right)^{\prime}
$$

At the same time from eq. (2) we obtain:

$$
w\left(x_{1}\right)=\frac{1}{2 R} x_{1}^{2} ; w\left(x_{1}\right)^{\prime}=\frac{x_{1}}{R}
$$

Combining together these equations we get:

$$
\frac{1}{R}=\frac{2 w\left(x_{2}\right)}{x_{1}\left(2 x_{2}-x_{1}\right)}
$$

The stress corresponding to this radius of curvature can be calculated by applying the equilibrium condition: the sum of all bending moments has to be zero. The stress induced by the piezoelectric effect $\sigma_{1}$ exerts the bending moment $M_{\mathrm{p}}$ :

$$
M_{\mathrm{p}}=-\sigma_{1} b_{\text {tel }} t_{\mathrm{p}} \frac{t_{\text {sub }}}{2}
$$

where $t_{\mathrm{p}}$ and $t_{\text {sub }}$ are the thicknesses of the piezoelectric layer and the substrate respectively. $b_{\text {tel }}$ represents the top electrode width: the piezoelectrically induced stress is indeed present only in the section of the film covered by the top electrode. As the residual stress of a film, the piezoelectrically induced stress is biaxial, meaning that the Stoney formula applies below the top electrode. There, the effective elastic modulus is then the biaxial modulus $Y_{\text {sub }} /\left(1-v_{\text {sub }}\right)$ [20], where $Y_{\text {sub }}$ and $v_{\text {sub }}$ are the substrate Young's modulus and Poisson's ratio respectively. The applicability of the Stoney equation in the cantilever structure cases has been extensively discussed by Chekchaki et al. [21]. The error with the dimensions used in our design are smaller than one percent. Especially helpful is the fact that we deal with $t_{\mathrm{p}} / t_{\text {sub }}<<1$, and a slender cantilever (width/length $<1$ ). According to the criteria given there, we are far away from bifurcations even at very large stresses. Theoretically, the threshold would be $3 \mathrm{GPa}$, which is far above for piezoelectric stresses usually limited to about $500 \mathrm{MPa}$ because of film cracking [22], [23]. For the bent borders outside the top electrode, the substrate reaction is instead described by the simple Young's modulus of the substrate $Y_{\text {sub }}$. The substrate then reacts to the piezoelectric actuation with a moment $M_{\text {sub }}$ equal to:

$$
M_{\text {sub }}=\int_{-t_{\text {sub }} / 2}^{+t_{\text {sub }} / 2}\left(\frac{Y_{\text {sub }}}{1-v_{\text {sub }}} b_{\text {tel }}+Y_{\text {sub }}\left(b_{\text {cant }}-b_{\text {tel }}\right)\right) \frac{z}{R} z d z
$$

where $b_{\text {cant }}$ is the cantilever width, and $z$ is the vertical distance from the neutral plane; which we can approximate to be in the middle of the substrate since we assumed a film thickness much smaller than the substrate's one. By applying the equilibrium condition $M_{\text {sub }}+M_{\mathrm{p}}=0$ combined with eq. (5) the following expression for the in-plane stress is obtained

$$
\begin{aligned}
\sigma_{1}= & \frac{1}{3} \frac{Y_{\text {sub }}}{\left(1-v_{\text {sub }}\right) c_{f}}\left[c_{f}+\left(1-c_{f}\right)\left(1-v_{\text {sub }}\right)\right] \\
& \frac{w\left(x_{2}\right)}{x_{1}\left(2 x_{2}-x_{1}\right)} \frac{t_{\text {sub }}^{2}}{t_{\mathrm{p}}}
\end{aligned}
$$

where with $c_{f}=b_{\text {tel }} / b_{\text {cant }}$ we indicate the top electrode coverage factor. There is therefore a linear relationship between the in-plane stress $\sigma_{1}$ and the measured cantilever 
tip displacement $w\left(x_{2}\right)$. From the definition of the effective transverse piezoelectric coefficient (eq. (1)) we get:

$$
\begin{aligned}
e_{31, f}= & -\frac{1}{3} \frac{Y_{\text {sub }}}{\left(1-v_{\text {sub }}\right) c_{f}}\left[c_{f}+\left(1-c_{f}\right)\left(1-v_{\text {sub }}\right)\right] \\
& \frac{w\left(x_{2}\right)}{x_{1}\left(2 x_{2}-x_{1}\right)} \frac{t_{\text {sub }}^{2}}{V}
\end{aligned}
$$

Where $V$ is the excitation voltage. For unpatterned top electrodes $\left(c_{f}=1\right.$ and $\left.x_{1}=x_{2}\right)$ eq. (9) coincide with what obtained by Kanno's group [18].

We have seen that for the determination of the in-plane stress, the knowledge of the substrate Young's modulus and Poisson's ratio are required. The very large majority of piezo-MEMS devices are obtained out of single-crystal silicon wafers: the mechanical properties of the sample are therefore anisotropic, i.e. they depend on the crystal cut and on the direction of the longitudinal axis of the beam. A didactic review of silicon elastic properties as a function of the crystal orientation can be found in [24]. For the most common situation, standard $\{100\}$ silicon wafers are employed, and the test beams are cut or cleaved most conveniently perpendicular or parallel to the primary flat, giving beams with the main axis along a $<110>$ direction. In this case the substrate Young's modulus and Poisson's ratio are respectively $169 \mathrm{GPa}$ and 0.064. All the samples studied in this paper are prepared with this geometry. This is unlike to ref. [16], where $<100>$ directions were chosen in order to use the textbook values for $Y(130.5 \mathrm{GPa})$ and $v(0.28)$.

\section{Test Measurements}

\section{A. Some Examples}

We present now typical examples of measurements performed on samples diced out of a $380 \mu \mathrm{m}$ thick $\{100\}$ silicon wafer, on top of which a $1.18 \mu \mathrm{m}$ thick PZT (53/47) thin film has been deposited with an established sol-gel method [25]-[27]. X-rays diffraction and scanning electron microscopy analysis (not shown) reveal a fiber textured (100)-oriented perovskite phase with dense and smooth microstructure. The platinum top electrode has been patterned by a lift-off procedure with DC sputtering at room temperature. The whole wafer has been then annealed in oxygen for ten minutes at $550{ }^{\circ} \mathrm{C}$. No poling procedure was applied. The analyzed samples are full-wafer thickness beams, $1.5 \mathrm{~mm}$ wide and $15 \mathrm{~mm}$ long. Four different samples with increasing top electrode length $(2 \mathrm{~mm}, 3 \mathrm{~mm}, 7 \mathrm{~mm}$ and $8 \mathrm{~mm})$ have been investigated with the displacement sensor placed at different positions. The electrode width is $1 \mathrm{~mm}$ in all cases and the unclamped part of the cantilever is $10 \mathrm{~mm}$ long.

In this case the optical probe version of the setup has been employed: the output noise level (equivalent to $2 \mathrm{~nm}$ excursion) corresponds to $0.03 \mathrm{MPa}$ of in-plane stress for the $8 \mathrm{~mm}$ long top electrode case. In Fig. 3 we can appreciate a typical large-signal response of the film subjected to sinusoidal bipolar excitation (zero DC offset) at $110 \mathrm{~Hz}$. In Fig. 4 smallsignal $e_{31, f}$ is depicted as a function of the DC bias, together with the simultaneously measured relative permittivity. The small signal excitation is at $0.66 \mathrm{kHz}$ and has an amplitude of

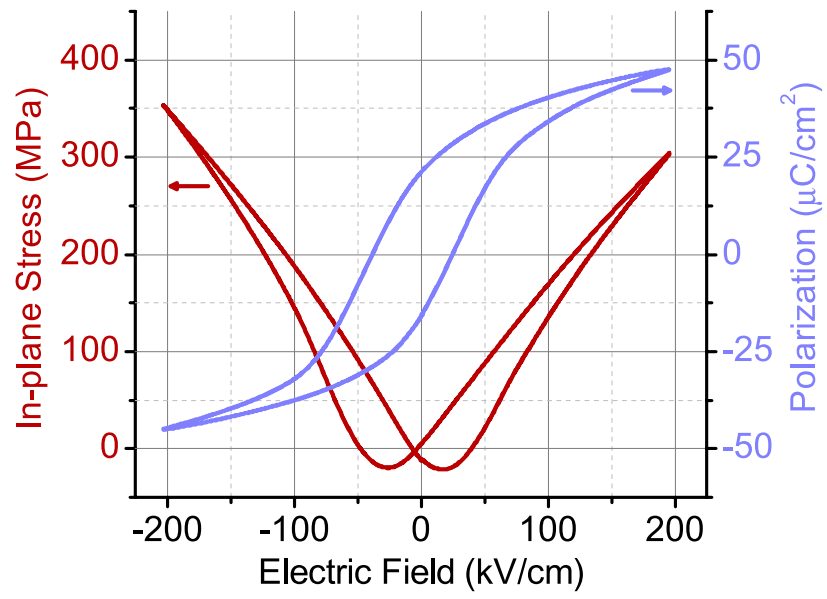

Fig. 3. Example of simultaneous measurement of mechanical and electrical large signal response of a sol-gel deposited PZT 53/47 thin film. The electric field was swept sinusoidally at a frequency of $110 \mathrm{~Hz}$.

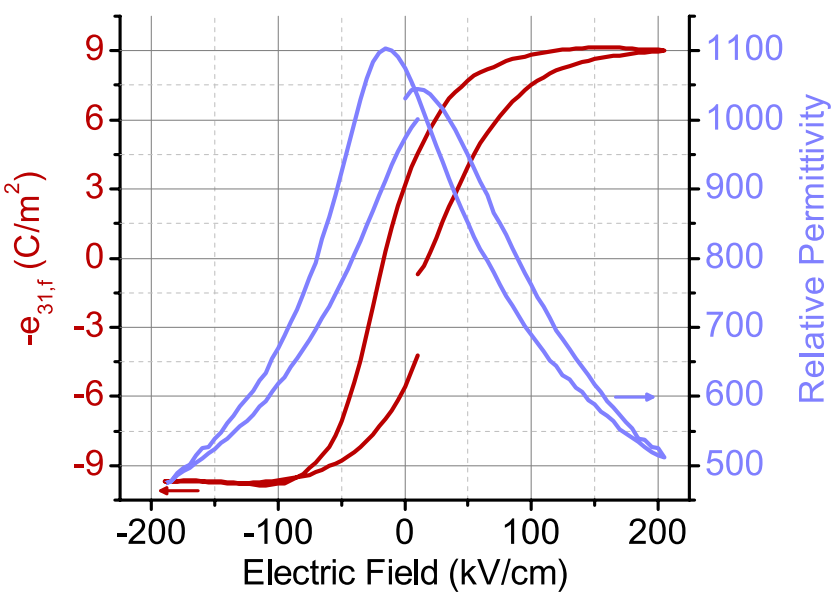

Fig. 4. Typical small-signal response of a sol-gel deposited PZT 53/47 thin film. $e_{31, f}$ and relative permittivity are measured simultaneously. The small signal excitation field has a frequency of $0.66 \mathrm{kHz}$ and an amplitude of $1 \mathrm{kV} / \mathrm{cm}$ RMS. The complete loop was measured in $480 \mathrm{~s}$.

$1 \mathrm{kV} / \mathrm{cm}$. In this paper we define the sign of the electric field positive when it is pointing towards the substrate (grounded bottom electrode and positive voltage applied to the top electrode).

\section{B. Experimental Validation of the Stress Formula}

Equation (8) was experimentally verified for various values of $x_{1}$ (by changing the cantilever sample) and $x_{2}$ (by sweeping the position of the displacement sensor). Assuming identical PZT thin film properties on all investigated beams, the same stress is generated at a given electric field, and identical curvatures $1 / R$ are obtained. The beam deflection $w\left(x_{2}\right)$ is then a linear function of $x_{1}\left(2 x_{2}-x_{1}\right)$. Results are depicted in Fig. 5. The linear fit is excellent in all cases, proving that eq. (8) is compatible with the cantilever behavior, and that the uniformity of piezoelectric properties across the wafer is good.

The direct and the converse piezoelectric coefficients are identical in theory [28], but in practice, ferroelectric thin films often show different values as measurement conditions and prehistory (e.g. poling) are different [29]. For non-ferroelectric thin films, like AlN, the polarization cannot be changed under 


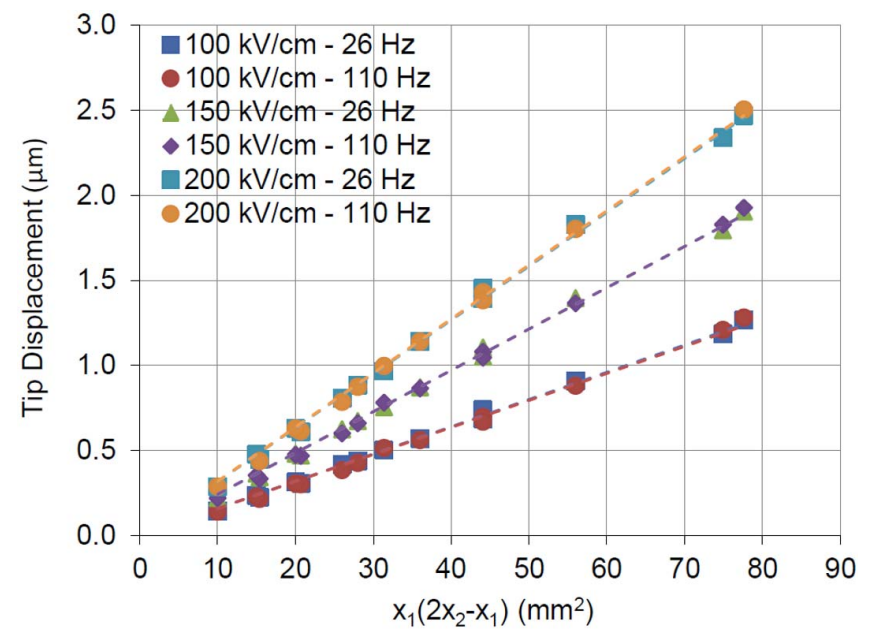

Fig. 5. Cantilever tip displacement $w\left(x_{2}\right)$ as a function of the geometrical parameter $x_{1}\left(2 x_{2}-x_{1}\right)$ for different fields and frequencies. Four cantilevers with different values of $x_{1}(2 \mathrm{~mm}, 3 \mathrm{~mm}, 7 \mathrm{~mm}$ and $8 \mathrm{~mm})$ have been investigated at different probe positions $x_{2}$ and under different excitation conditions. Their sequence has been kept constant in order to ensure that the comparison is done between samples with the same history. The linear fit is excellent in all the cases: this means that the film properties are homogeneous along the wafer and that eq. (8) is compatible with the cantilever behavior.

TABLE I

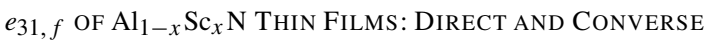
EFFECT MEASUREMENTS

\begin{tabular}{rcc}
\hline & $\begin{array}{c}\text { converse } e_{31, f}\left(\mathrm{C} / \mathrm{m}^{2}\right) \\
\text { this work (at } 110 \mathrm{~Hz})\end{array}$ & $\begin{array}{c}\text { direct } e_{31, f}\left(\mathrm{C} / \mathrm{m}^{2}\right) \\
\text { Matloub } \text { et al. }[31]\end{array}$ \\
\hline $\mathrm{AlN}$ & $-1.14 \pm 0.06$ & $-1.00 \pm 0.05$ \\
$\mathrm{Al}_{0.88} \mathrm{Sc}_{0.12} \mathrm{~N}$ & $-1.56 \pm 0.08$ & $-1.36 \pm 0.07$ \\
$\mathrm{Al}_{0.83} \mathrm{Sc}_{0.17} \mathrm{~N}$ & $-1.60 \pm 0.08$ & $-1.47 \pm 0.07$ \\
\hline
\end{tabular}

the application of an external stress or field, nor degrade with time. Aluminum nitride is therefore an ideal candidate for a comparison of this setup with the existing ones. In the past the effective transverse piezoelectric coefficient for this material has been measured as $e_{31, f}=-1.05 \mathrm{C} / \mathrm{m}^{2}$ from a transducer response [30], $-1.02 \mathrm{C} / \mathrm{m}^{2}$ from a charge/strain experiment [16], and $-1.00 \mathrm{C} / \mathrm{m}^{2}$ as well from another charge/strain measurement [31]. We performed small-signal measurements of $e_{31, f}$ for c-axis textured $\mathrm{AlN}$ and $\mathrm{Al}_{1-x} \mathrm{Sc}_{x} \mathrm{~N}$ thin films (see [31] for details) as a function of the frequency, by coupling the optical probe with a lock-in amplifier. Cantilevers with the same geometry as the ones used for PZT characterization have been prepared. The top electrode length amounted to $3 \mathrm{~mm}$. The results are summarized in Table I. The fixation of the cantilever to the clamping structure is facilitated by the presence of a groove in the supporting plate, which allows alignment of the top electrode with the bar with less than a $0.1 \mathrm{~mm}$ error. A similar tolerance is achieved for the displacement sensor positioning, resulting in a total uncertainty of about $5 \%$ for this case. The groove also defines the orthogonality of the beam with respect to the clamp: the very small angular deviations have negligible effects on the cantilever behavior, being ruled by a cosine law. The values measured for the converse effective piezoelectric coefficient are $8 \%$ to $13 \%$ higher than measured from the charge/strain response. This discrepancy is within the estimated alignment

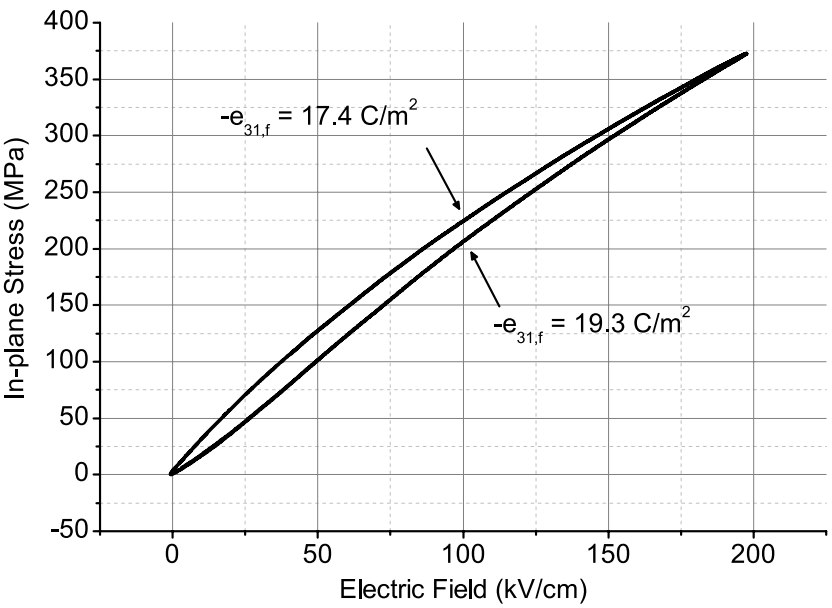

Fig. 6. Large signal piezoelectric response to a sinusoidal unipolar $(90 \mathrm{~Hz})$ excitation of a "gradient-free" PZT (53/47) thin film. The numerical differentiation of the curve gives the $e_{31, f}$ value, indicated for $100 \mathrm{kV} / \mathrm{cm}$.

errors. Nevertheless, it looks rather systematic, and probably comes from not so well defined positioning of the actuator in the set up for the direct effect. At least for the case of AlN, the average value of $-1.07 \mathrm{C} / \mathrm{m}^{2}$ agrees well with the literature value of $-1.05 \mathrm{C} / \mathrm{m}^{2}[30]$.

\section{Device-Like Excitation of PZT Thin Films}

The real advantage of this characterization method lies in the capability of tracking the piezoelectric response of a thin film under driving voltages of the application. The majority of the devices of interest have membranes structures, actuated with large signal unipolar voltages, in order to avoid ferroelectric fatigue and increase the linearity of the operation. Fig. 6 shows the in-plane stress response as a function of the electric field for a unipolar, sinusoidal excitation $V(t)=A(1+\sin (2 \pi f t))$ at $90 \mathrm{~Hz}$. The analyzed sample is a "gradient-free" PZT (53/47) thin film deposited with an optimized sol-gel route described in details elsewhere [32]. The film is highly (100)-textured and $1.17 \mu \mathrm{m}$ thick. The platinum top electrode has been patterned by lift-off through room temperature sputtering. The whole wafer has been subsequently annealed in oxygen for ten minutes at $550{ }^{\circ} \mathrm{C}$. Values for the effective transverse piezoelectric coefficient are then determined through a numerical differentiation of the in-plane stress with respect to the electric field according to eq. (1). At $100 \mathrm{kV} / \mathrm{cm}$, we obtain $-19.3 \mathrm{C} / \mathrm{m}^{2}$ with increasing and $-17.4 \mathrm{C} / \mathrm{m}^{2}$ with decreasing electric field. The average value of $-18.3 \mathrm{C} / \mathrm{m}^{2}$ is then representative of the sample behavior. The value in the direct mode at zero electric field is $30 \%$ lower, ${ }^{1}$ due to a smaller remanent polarization as compared to the converse mode, in which the unipolar cycling refreshes $P$ constantly. For this film $e_{31, f}$ (i.e. the slope of the graph in Fig. 6) does not degrade under the application of large electric fields, compatibly with what is expected from its dielectric analysis (not shown) which reveals that the product of $P(E) \cdot \varepsilon(E)$ is roughly constant for $E>100 \mathrm{kV} / \mathrm{cm}$, like $e_{31, f}$. Despite we deal with a polycrystalline multidomain

\footnotetext{
${ }^{1}$ for this film we recorded $(-12.6 \pm 0.06) \mathrm{C} / \mathrm{m}^{2}$, after poling at $150{ }^{\circ} \mathrm{C}$ with a DC field of $150 \mathrm{kV} / \mathrm{cm}$ for 10 minutes.
} 


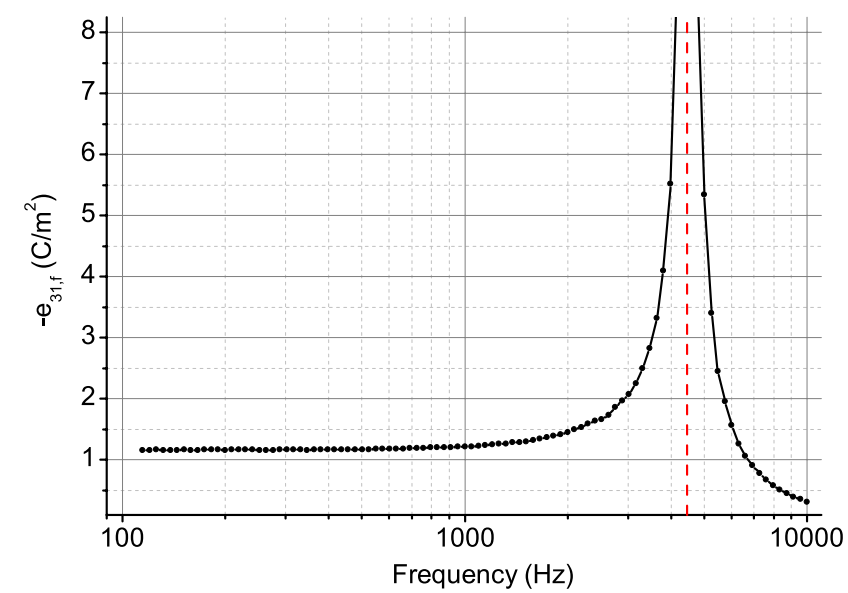

Fig. 7. Frequency dependence of the small-signal converse $e_{31, f}$ coefficient for the AlN film. An electro-mechanical resonance is observed at $4.4 \mathrm{kHz}$ The $y$-axis of the graph is limited by the saturation of the lock-in amplifier.

system, this behavior corresponds to what is derived from the free energy of the single domain state, for which the piezoelectric coefficients are indeed proportional to $P \cdot \varepsilon$ [33].

\section{Limitations of the Measurement Technique}

For any AC measurement technique, the presence of resonances might lead to severe artifacts in the evaluation of the piezoelectric coefficient. The excitation frequency should be chosen far away from any eigenfrequencies of the analyzed structure. The fundamental resonance of the cantilevers used in this work was found at $4.4 \mathrm{kHz}$ in the $e_{31, f}$ response (Fig. 7). The resonance enhancement is larger than one order of magnitude.

\section{Reliability of PZT Thin Films}

This setup can be used to investigate the reliability of cantilever samples, i.e. the endurance of the piezoelectric and ferroelectric response for a given excitation amplitude and waveform. Here we report the results of a test made on a "gradient-free" PZT based cantilever respect to excitations of $-150 \mathrm{kV} / \mathrm{cm}$. The fatigue treatment signal is a unipolar square waveform, with a duty cycle of $50 \%$ and a frequency of $100 \mathrm{kHz}$; which corresponds to a period that is ten times longer than the measured RC constant of the device, thus ensuring that the full voltage is effectively applied to the piezoelectric capacitor. The response was tested up to $10^{9}$ actuations: every decade, three measurements of cantilever tip displacement and polarization loop were recorded with an aixACCT TF2000 analyzer coupled with the SIOS singlelaser beam interferometer. The test waveform was unipolar of triangular shape with a cycle time of $0.5 \mathrm{~s}$, having as well a peak-to-peak amplitude of $-150 \mathrm{kV} / \mathrm{cm}$. The sample response before the endurance test is depicted in Fig. 8. Note that only polarization variations $\Delta P$ can be measured from the electric current response. In Fig. 9 we report the maximum cantilever tip displacement and the maximum polarization variation $\Delta P$ as a function of the number of excitation cycles. While there is no relevant degradation of the piezoelectric activity of the film, we observe changes in the polarization behavior.

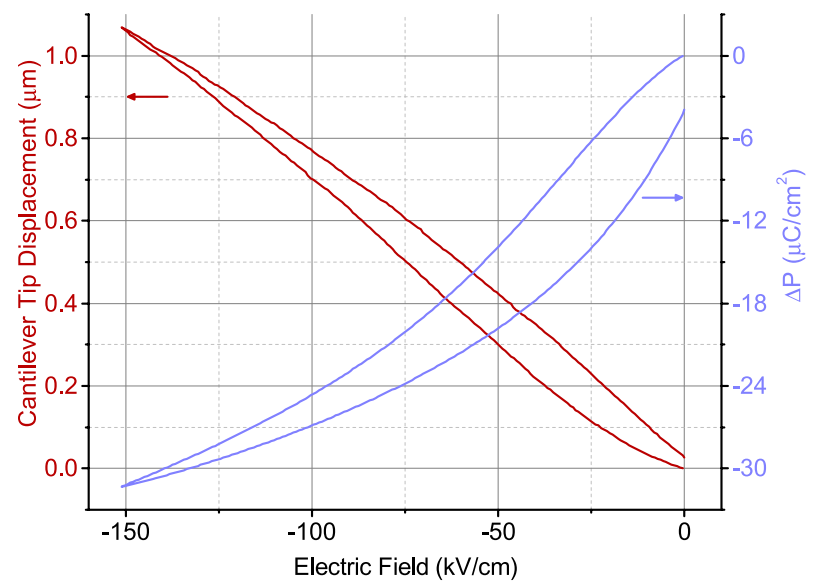

Fig. 8. Cantilever response before the endurance test. The analyzed sample is a PZT 53/47 "gradient-free" film, with a $3 \mathrm{~mm}$ long top electrode. The laser spot was at $8.85 \mathrm{~mm}$ away from the clamp.

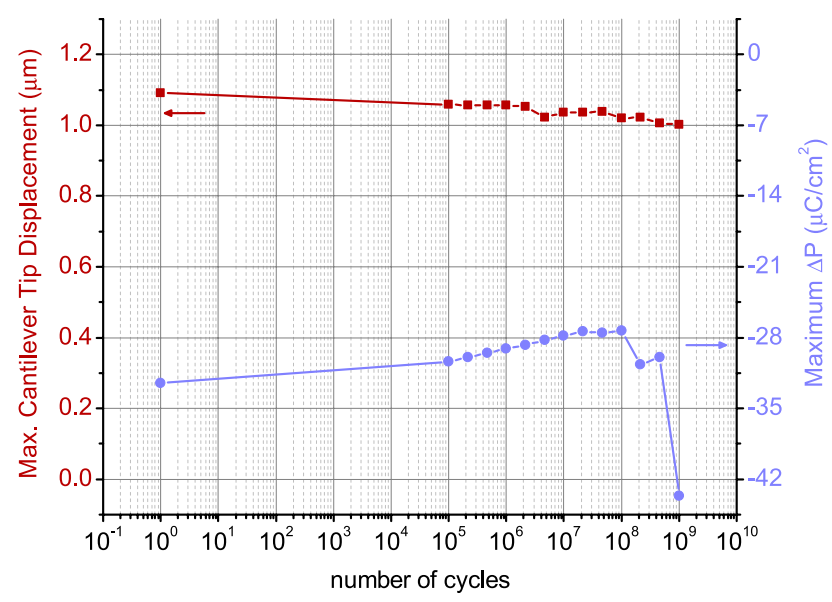

Fig. 9. Maximum polarization variation and cantilever tip displacement as a function of the number of excitation cycles for the PZT 53/47 "gradient-free" film.
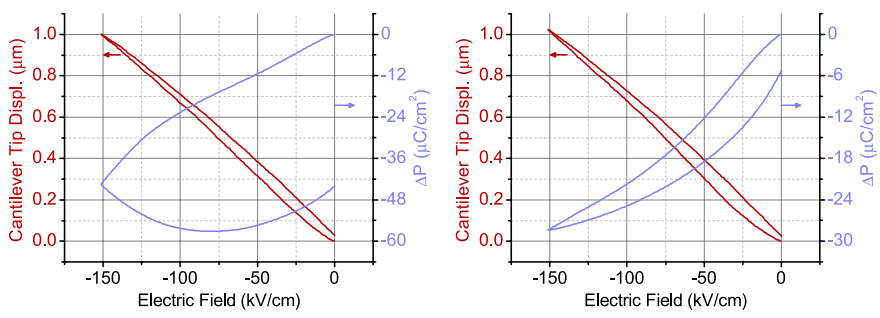

Fig. 10. Response obtained at the end of (left), and ten minutes after (right) the endurance test for the PZT 53/47 "gradient-free" film.

The measurements shown in Fig. 10 clearly show that the polarization change is due to leakage (charging is proportional to the applied voltage above about $-100 \mathrm{kV} / \mathrm{cm}$ ), and no noticeable changes in the displacement curve are observed. The leakage was not large enough to reduce the applied voltage significantly and turned out to be quite reversible: ten minutes after the end of the endurance test, the polarization change loop returned to almost the initial one.

Several mechanisms can be responsible for a leakage increase during the fatigue test, most likely charge injection or temperature increase. Thermal conduction and charge diffusion are enough fast to explain the relaxation into the initial stage 
within minutes. Heat is indeed generated by the hysteresis of the P-V loop. The area comprised between the up and the down branch is the heat dissipated into the film with every cycle. We calculated that this value is around $1.77 \mu \mathrm{J}$ for the first loop. As we cycle with $100 \mathrm{kHz}$, the power input amounts to $0.177 \mathrm{~W}$. The beam possesses a very small heat capacity of only $14 \mathrm{~mJ} / \mathrm{K}$ (taking into the account the heat capacity of silicon only) and therefore the temperature might increase by $12 \mathrm{~K} / \mathrm{s}$. This effect is reduced by thermal conduction through the silicon beam, which is a very good thermal conductor. If the clamped end is at constant room temperature, the temperature increase would be limited to about $22 \mathrm{~K}$. We found a modest temperature increase of $3 \mathrm{~K}$ at the clamped end to which we fixed a Pt-100 temperature resistor, proving the heat production on the beam. The leaky P-V loop at the end of the fatigue tests produces instead a heat dissipation of roughly $1 \mathrm{~W}$, and therefore the temperature increase might reach $100 \mathrm{~K}$. We deal then with a typical runaway effect: the higher the temperature, the higher the losses, and the higher the losses, the higher the dissipated power heating up the cantilever. This shows that for accelerated tests one should keep the temperature under control by cooling. We remind the reader that the results obtained in the full wafer thickness case represent one end of the parameter field, where stresses are maximal but strains are minimal. The latter are indeed equal to the quantity $-t_{\text {sub }} / 2 R$, and in our case they are in the $10^{-5}$ range: 100 times less than what is normally achieved by unclamped PZT systems. In the full-wafer thickness case the mechanical stress will prevail, and thus cracking of the film might be the highest risk; while films embedded in thin micromachined structures might show different fatigue and/or failure mechanisms. When dealing with thinner structures, the effect of mechanical clamping is reduced [34] and ferroelastic domains might change their dimensions and orientations more easily. As a consequence, the hysteresis loop might look differently as well, eventually contributing to higher losses during cycling. Also heat evacuation through a thin membrane will be lower, thus concentrating more heat in the PZT structure.

\section{CONCLUSIONS}

We developed and tested a versatile setup to evaluate the piezoelectrically induced in-plane stress of a thin film under the application of an arbitrary waveform. Both small signal and large signal tests can be performed correlating simultaneously measured in-plane piezoelectric coefficient with the dielectric response as a function of sweeping DC electric field, or the in-plane stress with the change in polarization, always as a function of the electric field. We applied the method to evaluate the response of sol-gel deposited PZT thin films for both bipolar and unipolar sinusoidal excitation. With this technique, the extent of non-linearities in the piezoelectric response and the dissipated energy are readily evaluated. We proposed to define the $e_{31, f}$ for piezo-MEMS actuators as the derivative of the stress curve at half of the maximum excitation field. Up and down values should be given to get an idea of the hysteresis. In addition we performed a reliability test on a "gradient free" PZT thin film: its converse piezoelectric activity is not degraded in a significant way up to $10^{9}$ actuations, a performance compatible with what was reported for the direct effect by Polcawich and TrolierMcKinstry [35] for a PZT 52/48 thin film. Nevertheless, we observed an increase of the leakage losses towards the end of the the endurance test. The relative fast self-recovery after the testing hints to a thermal runaway mechanism, initiated by the heat dissipated by the ferroelectric hysteresis, eventually reinforced by charge injection, and finally leading to electrical leakage.

\section{ACKNOWLEDGMENTS}

The authors would like to thank aixACCT Systems GmbH for the TF2000 Analyzer tool and M. Hadad for the help in the $\mathrm{Al}_{1-x} \mathrm{Sc}_{x} \mathrm{~N}$ thin film depositions.

\section{REFERENCES}

[1] J. Brunahl and A. M. Grishin, "Piezoelectric shear mode drop-ondemand inkjet actuator," Sens. Actuators A, Phys., vol. 101, no. 3, pp. 371-382, 2002.

[2] D. L. Polla and L. F. Francis, "Processing and characterization of piezoelectric materials and integration into microelectromechanical systems," Annu. Rev. Mater. Sci., vol. 28, pp. 563-597, Aug. 1998.

[3] P. Muralt et al., "Fabrication and characterization of PZT thin-film vibrators for micromotors," Sens. Actuators A, Phys., vol. 48, no. 2, pp. 157-165, 1995.

[4] R. Ried, E. Kim, D. Hong, and R. Mueller, "Piezoelectric microphone with on-chip CMOS circuits," J. Microelectromech. Syst., vol. 2, no. 3, pp. 111-120, 1993.

[5] N. Ledermann, P. Muralt, J. Baborowski, M. Forster, and J.-P. Pellaux, "Piezoelectric $\mathrm{Pb}\left(\mathrm{Zr}_{x}, \mathrm{Ti}_{1-x}\right) \mathrm{O}_{3}$ thin film cantilever and bridge acoustic sensors for miniaturized photoacoustic gas detectors," J. Micromech. Microeng., vol. 14, no. 12, pp. 1650-1658, 2004.

[6] B. Belgacem, F. Calame, and P. Muralt, "Piezoelectric micromachined ultrasonic transducers with thick PZT sol gel films," J. Electroceram., vol. 19, no. 4, pp. 369-373, 2007.

[7] R. Guerre et al., "Wafer-level transfer technologies for PZT-based RF MEMS switches," J. Microelectromech. Syst., vol. 19, no. 3, pp. 548-560, 2010.

[8] R. Proie, R. Polcawich, J. Pulskamp, T. Ivanov, and M. Zaghloul, "Development of a PZT MEMS switch architecture for low-power digital applications," J. Microelectromech. Syst., vol. 20, no. 4, pp. 1032-1042, 2011.

[9] C. Rhee, J. S. Pulskamp, R. G. Polcawich, and K. R. Oldham, "Multidegree-of-freedom thin-film PZT-actuated microrobotic leg," J. Microelectromech. Syst., vol. 21, no. 6, pp. 1492-1503, 2012.

[10] U. Baran et al., "Resonant PZT MEMS scanner for high-resolution displays," J. Microelectromech. Syst., vol. 21, no. 6, pp. 1303-1310, 2012.

[11] J. R. Bronson, J. S. Pulskamp, R. G. Polcawich, C. M. Kroninger, and E. D. Wetzel, "PZT MEMS actuated flapping wings for insectinspired robotics," in Proc. IEEE 22nd Int. Conf. Microelectromech. Syst., Jan. 2009, pp. 1047-1050.

[12] P. Muralt, R. G. Polcawich, and S. Trolier-McKinstry, "Piezoelectric thin films for sensors, actuators, and energy harvesting," MRS Bull., vol. 34, no. 9, pp. 658-664, 2009.

[13] P. Muralt, A. Kholkin, M. Kohli, and T. Maeder, "Piezoelectric actuation of PZT thin-film diaphragms at static and resonant conditions," Sens. Actuators A, Phys., vol. 53, nos. 1-3, pp. 398-404, 1996.

[14] J. F. Shepard, P. J. Moses, and S. Trolier-McKinstry, "The wafer flexure technique for the determination of the transverse piezoelectric coefficient $\left(d_{31}\right)$ of PZT thin films," Sens. Actuators A, Phys., vol. 71, nos. 1-2, pp. 133-138, 1998.

[15] R. H. T. Wilke, P. J. Moses, P. Jousse, C. Yeager, and S. Trolier-McKinstry, "Wafer mapping of the transverse piezoelectric coefficient, $e_{31, f}$, using the wafer flexure technique with sputter deposited Pt strain gauges," Sen. Actuators A, Phys., vol. 173, no. 1, pp. 152-157, 2012.

[16] M.-A. Dubois and P. Muralt, "Measurement of the effective transverse piezoelectric coefficient $e_{31, f}$ of $\mathrm{AlN}$ and $\mathrm{Pb}\left(\mathrm{Zr}_{x}, \mathrm{Ti}_{1-x}\right) \mathrm{O}_{3}$ thin films," Sens. Actuators A, Phys., vol. 77, no. 2, pp. 106-112, 1999. 
[17] K. Prume, P. Muralt, F. Calame, T. Schmitz-Kempen, and S. Tiedke, "Piezoelectric thin films: Evaluation of electrical and electromechanical characteristics for MEMS devices," IEEE Trans. Ultrason., Ferroelectr, Freq. Control, vol. 54, no. 1, pp. 8-14, Jan. 2007.

[18] D.-M. Chun, M. Sato, and I. Kanno, "Precise measurement of the transverse piezoelectric coefficient for thin films on anisotropic substrate," J. Appl. Phys., vol. 113, no. 4, p. 044111, 2013.

[19] R. O. Cook and C. W. Hamm, "Fiber optic lever displacement transducer," Appl. Opt., vol. 18, no. 19, pp. 3230-3241, 1979.

[20] M. Ohring, Materials Science of Thin Films. New York, NY, USA: Academic, 2002, ch. 12, pp. 727-730.

[21] M. Chekchaki, V. Lazarus, and J. Frelat, "Analytical and 3D finite element study of the deflection of an elastic cantilever bilayer plate," J. Appl. Mech., vol. 78, no. 1, p. 011008, 2011.

[22] K. Mehta and A. V. Virkar, "Fracture mechanisms in ferroelectricferroelastic lead zirconate titanate (zr: Ti=0.54:0.46) ceramics," J. Amer. Ceram. Soc., vol. 73, no. 3, pp. 567-574, 1990.

[23] S. L. dos Santos e Lucato, D. C. Lupascu, and J. Rödel, "Effect of poling direction on $R$-curve behavior in lead zirconate titanate," J. Amer. Ceram. Soc., vol. 83, no. 2, pp. 424-426, 2000.

[24] M. Hopcroft, W. Nix, and T. Kenny, "What is the Young's modulus of silicon?" J. Microelectromech. Syst., vol. 19, no. 2, pp. 229-238, 2010.

[25] K. D. Budd, S. K. Dey, and D. A. Payne, "Sol-gel processing of PT, PZ, PZT and PLZT thin films," Brit. Ceram. Proc., no. 36, pp. 107-121, 1985.

[26] S. Gurkovich and J. Blum, Preparation of Monolithic Lead-Titanate by Sol-Gel Process. Hoboken, NJ, USA: Wiley, 1984, pp. 152-160.

[27] N. Ledermann et al., " $\{100\}$-textured, piezoelectric $\mathrm{Pb}\left(\mathrm{Zr}_{x} \mathrm{Ti}_{1-x}\right) \mathrm{O}_{3}$ thin films for MEMS: Integration, deposition and properties," Sens. Actuators A, Phys., vol. 105, no. 2, pp. 162-170, 2003.

[28] M. Lines and A. Glass, Principles and Applications of Ferroelectrics and Related Materials, W. Marshall and D. Wilkinson, Eds. Oxford, U.K.: Clarendon, 1977.

[29] D. Damjanovic, "Ferroelectric, dielectric and piezoelectric properties of ferroelectric thin films and ceramics," Rep. Prog. Phys., vol. 61, no. 9, pp. 1267-1324, 1998

[30] K. Tsubouchi and N. Mikoshiba, "Zero-temperature-coefficient SAW devices on AlN epitaxial-films," IEEE Trans. Sonics Ultrason., vol. 32, no. 5, pp. 634-644, Sep. 1985.

[31] R. Matloub et al., "Piezoelectric $\mathrm{Al}_{1-x} \mathrm{Sc}_{x} \mathrm{~N}$ thin films: A semiconductor compatible solution for mechanical energy harvesting and sensors," Appl. Phys. Lett., vol. 102, no. 15, p. 152903, 2013.

[32] F. Calame and P. Muralt, "Growth and properties of gradient free solgel lead zirconate titanate thin films," Appl. Phys. Lett., vol. 90, no. 6, p. 062907, 2007.

[33] F. J. Haun, E. Furman, S. J. Jang, and L. E. Cross, "Thermodynamic theory of the lead zirconate-titanate solid-solution system. Part I: Phenomenology," Ferroelectrics, vol. 99, no. 1, pp. 13-25, 1989.

[34] F. Griggio et al., "Substrate clamping effects on irreversible domain wall dynamics in lead zirconate titanate thin films," Phys. Rev. Lett., vol. 108, no. 5, p. 157604, 2012.

[35] R. G. Polcawich and S. Trolier-McKinstry, "Piezoelectric and dielectric reliability of lead zirconate titanate thin films," J. Mater. Res., vol. 15, no. 11 , pp. $2505-2513,2000$

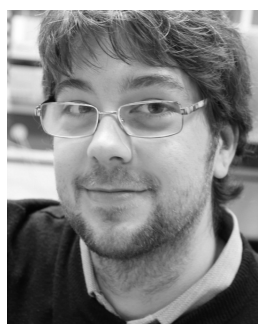

Andrea Mazzalai received the M.Sc. (Hons.) degree in physics of matter from the University of Trento, Trento, Italy, in 2008, with an experimental thesis on infrared spectroscopy of supersonic molecular beams. After a stay in 2009 at the international free-electron laser facility CLIO at Paris Sud XI University, Orsay, France, he is currently pursuing the Ph.D. degree with the Ceramics Laboratory, Swiss Federal Institute of Technology, Lausanne, Switzerland. His research activities are focused in the piezo- microelectromechanical systems technology, in particular, PZT thin-film processing by RF magnetron sputtering, piezoelectric materials characterization, and vibration energy harvesting.

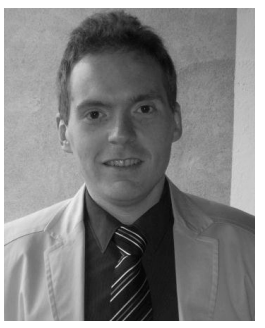

Davide Balma received the M.Sc. degree in physics of advanced technologies from the University of Turin, Turin, Italy, in 2008. He did his master's project at the National Institute of Metrological Research, Turin, working on magnetocaloric effect on magnetic shape memory alloys. He received the Ph.D. degree in electronic devices from the Polytechnic of Turin, Turin, in 2011, where he did his research activity mainly at the Materials and Microsystems Laboratory. During this period, he spent part of his time at the Swiss Federal Institute of Technology (EPFL), Lausanne, Switzerland. His main activity was the fabrication of a piezoelectrically actuated microswitch and sol-gel PZT deposition optimization. Since 2012, he has been a Post-Doctoral Scientist with EPFL, and his research interests include microelectromechanical systems fabrication technologies and piezoelectric materials (mainly sol-gel PZT) and devices.

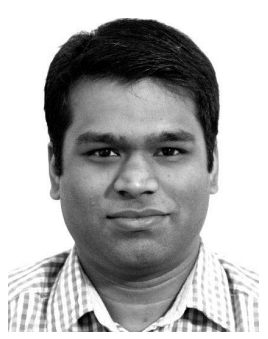

Nachiappan Chidambaram received the master's degree in materials engineering from the Indian Institute of Science, Bangalore, India, in 2009. His master's thesis was on ultrahigh-temperature ceramics processed by reactive hot pressing. He is currently pursuing the $\mathrm{Ph} . \mathrm{D}$. degree with the Ceramics Laboratory, Swiss Federal Institute of Technology, Lausanne, Switzerland. His Ph.D. thesis mainly focuses on sol-gel processing of PZT films.

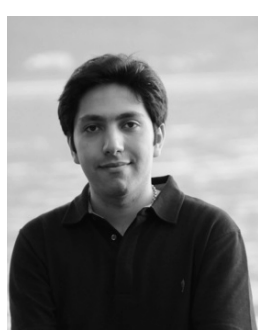

Ramin Matloub received the M.Sc. degree in nanotechnology from the Royal Institute of Technology, Stockholm, Sweden, in 2009. He did his master's project at the Federal Laboratories for Materials Science and Testing, Zurich, Switzerland. The project was mainly on electrical and magnetic properties of complex oxide films. He is currently pursuing the Ph.D. degree with the Swiss Federal Institute of Technology, Lausanne, Switzerland. He is currently working on AlScN thin films for energy harvesting and microwave filter applications.

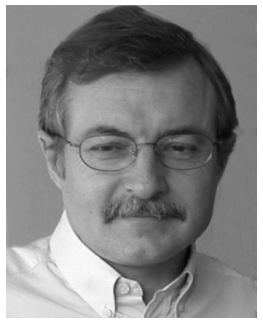

Paul Muralt (F'13) is currently an Adjunct Professor with the Swiss Federal Institute of Technology (EPFL), Lausanne, Switzerland. He leads a group in piezoelectric thin film, microelectromechanical systems, and nanotechnology activities at the Ceramics Laboratory, Materials Science Institute, EPFL. He has studied solid-state physics and accomplished a Ph.D. work in incommensurate structures at the ETH Zurich, Zurich, Switzerland. From 1984 to 1986, he was a Post-Doctoral Fellow with the IBM Research Laboratory, Zurich, where he pioneered the application of scanning tunneling microscopy to the imaging of the electrical surface potential. After a stay at the Free University of Berlin, Berlin, Germany, and several years as the Manager in a thin-film coating tool company, he joined EPFL in 1993. His main competences are in piezoelectric thin-film processing of perovskite ferroelectrics and polar films, such as AlN, in combination with piezoelectric micro and nano devices, covering particularly ultrasonic applications in the $\mathrm{MHz}$ to $\mathrm{GHz}$ range. More recent works also deal with oxygen ion conductors for microsolid oxide fuel cells. As a Teacher, he gives lectures in thin-film deposition, micro and nanotechnology, surface analysis, and basic ceramics for engineers. He has authored or co-authored over 200 scientific articles, which are cited over 6000 times in total. He is a member of the Materials Research Society and the American Ceramic Society. He was the Co-Chair of the 2008 MRS Spring Meeting, co-organized three MRS or E-MRS symposia, and was also a Program Committee Member of the specialized ferroelectric meetings ISAF 2007, ISIF 2008, and IFCS 2010. 